\title{
Condoms and STDs:
}

\section{Fact Sheet for Public Health Personnel}

Consistent and correct use of latex condoms can reduce (though not eliminate) the risk of STD transmission. To achieve the maximum protective effect, condoms must be used both consistently and correctly. Inconsistent use can lead to STD acquisition because transmission can occur with a single act of intercourse with an infected partner. Similarly, if condoms are not used correctly, the protective effect may be diminished even when they are used consistently. The most reliable ways to avoid transmission of sexually transmitted diseases (STDs), including human immunodeficiency virus (HIV), are to abstain from sexual activity or to be in a long-term mutually monogamous relationship with an uninfected partner. However, many infected persons may be unaware of their infections because STDs are often asymptomatic or unrecognized.

This fact sheet presents evidence concerning the condoms and the prevention of STDs, including HIV, based on information about how different STDs are transmitted, the physical properties of condoms, the anatomic coverage or protection that condoms provide, and epidemiologic studies assessing condom use and STD risk. This fact sheet updates previous CDC fact sheets on condom effectiveness for STD prevention by incorporating additional evidence-based findings from published epidemiologic studies. For more information on how to use a condom correctly, visit $\mathrm{CDC}$ 's Condom Effectiveness web site.

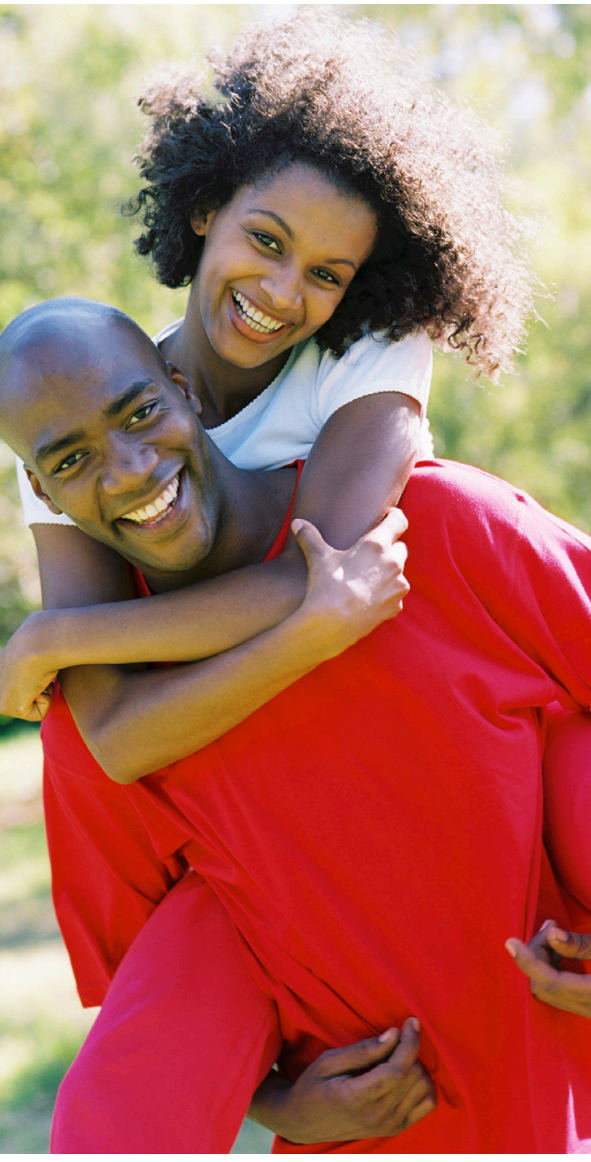

\section{Sexually Transmitted Diseases, Including HIV Infection}

Latex condoms, when used consistently and correctly, are highly effective in preventing the sexual transmission of HIV, the virus that causes AIDS. In addition, consistent and correct use of latex condoms reduces the risk of other sexually transmitted diseases (STDs), including diseases transmitted by genital secretions, and to a lesser degree, genital ulcer diseases. Condom use may reduce the risk for genital human papillomavirus (HPV) infection and HPV-associated diseases, e.g., genital warts and cervical cancer.

There are two primary ways that STDs are transmitted. Some diseases, such as HIV infection, gonorrhea, chlamydia, and trichomoniasis, are transmitted when infected urethral or vaginal secretions contact mucosal surfaces (such as the male urethra, the vagina, or cervix). In contrast, genital ulcer diseases (such as genital herpes, syphilis, and chancroid) and human papillomavirus (HPV) infection are primarily transmitted through contact with infected skin or mucosal surfaces.

Laboratory studies have demonstrated that latex condoms provide an essentially impermeable barrier to particles the size of STD pathogens.

Theoretical and empirical basis for protection. Condoms can be expected to provide different levels of protection for various STDs, depending on differences in how the diseases are transmitted. Condoms block transmission and acquisition of STDs by preventing contact between the condom wearer's penis and a sex partner's skin, mucosa, and genital secretions. A greater level of protection is provided for the diseases transmitted by genital secretions. A lesser degree of protection is provided for genital ulcer diseases or HPV because these infections also may be transmitted by exposure to areas (e.g., infected skin or mucosal surfaces) that are not covered or protected by the condom.

Epidemiologic studies seek to measure the protective effect of condoms by comparing risk of STD transmission among condom users with nonusers who are engaging in sexual intercourse. Accurately estimating the effectiveness of condoms for prevention of STDs, 


\section{Other Diseases transmitted by genital secretions, including Gonorrhea, Chlamydia, and Trichomoniasis}

Latex condoms, when used consistently and correctly, reduce the risk of transmission of STDs such as gonorrhea, chlamydia, and trichomoniasis.

STDs such as gonorrhea, chlamydia, and trichomoniasis are sexually transmitted by genital secretions, such as urethral or vaginal secretions.

Laboratory studies have demonstrated that latex condoms provide an essentially impermeable barrier to particles the size of STD pathogens.

Theoretical basis for protection. The physical properties of latex condoms protect against diseases such as gonorrhea, chlamydia, and trichomoniasis by providing a barrier to the genital secretions that transmit STD-causing organisms.

Epidemiologic studies that compare infection rates among condom users and nonusers provide evidence that latex condoms can protect against the transmission of STDs such as chlamydia, gonorrhea and trichomoniasis.

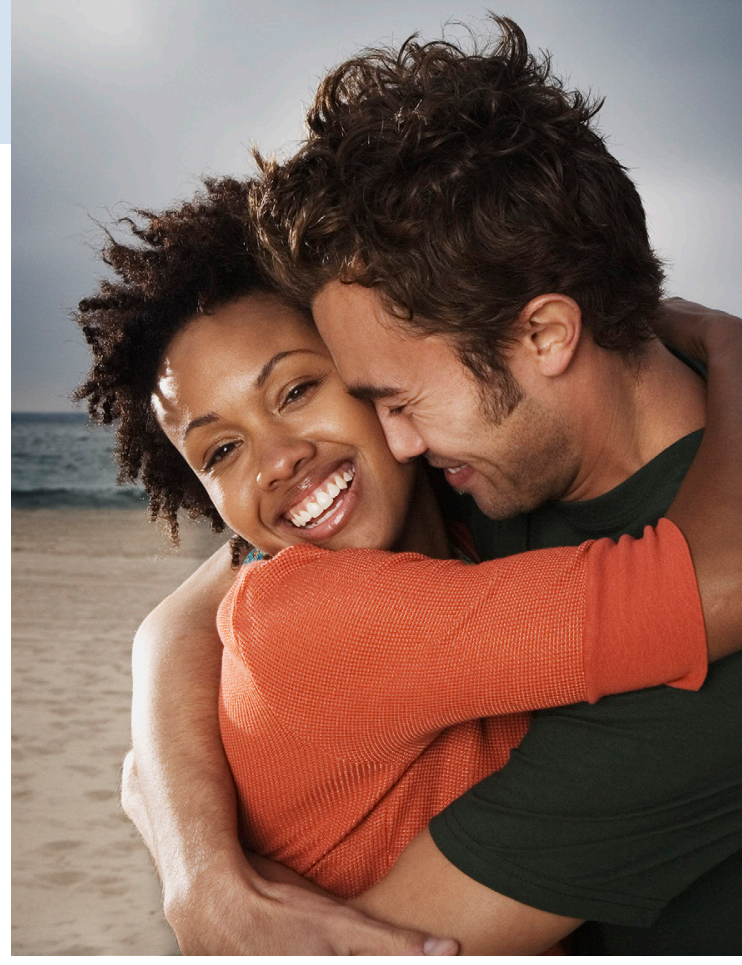

\section{Genital ulcer diseases and HPV infections}

Genital ulcer diseases and HPV infections can occur in both male and female genital areas that are covered or protected by a latex condom, as well as in areas that are not covered. Consistent and correct use of latex condoms reduces the risk of genital herpes, syphilis, and chancroid only when the infected area or site of potential exposure is protected. Condom use may reduce the risk for HPV infection and HPV-associated diseases (e.g., genital warts and cervical cancer).

Genital ulcer diseases include genital herpes, syphilis, and chancroid. These diseases are transmitted primarily through "skin-to-skin" contact from sores/ulcers or infected skin that looks normal. HPV infections are transmitted through contact with infected genital skin or mucosal surfaces/secretions. Genital ulcer diseases and HPV infection can occur in male or female genital areas that are covered (protected by the condom) as well as those areas that are not.

Laboratory studies have demonstrated that latex condoms provide an essentially impermeable barrier to particles the size of STD pathogens.

Theoretical basis for protection. Protection against genital ulcer diseases and HPV depends on the site of the sore/ulcer or infection. Latex condoms can only protect against transmission when the ulcers or infections are in genital areas that are covered or protected by the condom. Thus, consistent and correct use of latex condoms would be expected to protect against transmission of genital ulcer diseases and HPV in some, but not all, instances.
Epidemiologic studies that compare infection rates among condom users and nonusers provide evidence that latex condoms provide limited protection against syphilis and herpes simplex virus-2 transmission. No conclusive studies have specifically addressed the transmission of chancroid and condom use, although several studies have documented a reduced risk of genital ulcers associated with increased condom use in settings where chancroid is a leading cause of genital ulcers.

Condom use may reduce the risk for HPV-associated diseases (e.g., genital warts and cervical cancer) and may mitigate the other adverse consequences of infection with HPV; condom use has been associated with higher rates of regression of cervical intraepithelial neoplasia (CIN) and clearance of HPV infection in women, and with regression of HPV-associated penile lesions in men. A limited number of prospective studies have demonstrated a protective effect of condoms on the acquisition of genital HPV.

While condom use has been associated with a lower risk of cervical cancer, the use of condoms should not be a substitute for routine screening with Pap smears to detect and prevent cervical cancer, nor should it be a substitute for HPV vaccination among those eligible for the vaccine Selected References are available at: www.cdc.gov/condomeffectiveness/references.html 